\title{
Cold Still Kills: Cold-Related Illnesses In Military Practice Freezing And Non-Freezing Cold Injury
}

\section{CHE Imray, EHN Oakley}

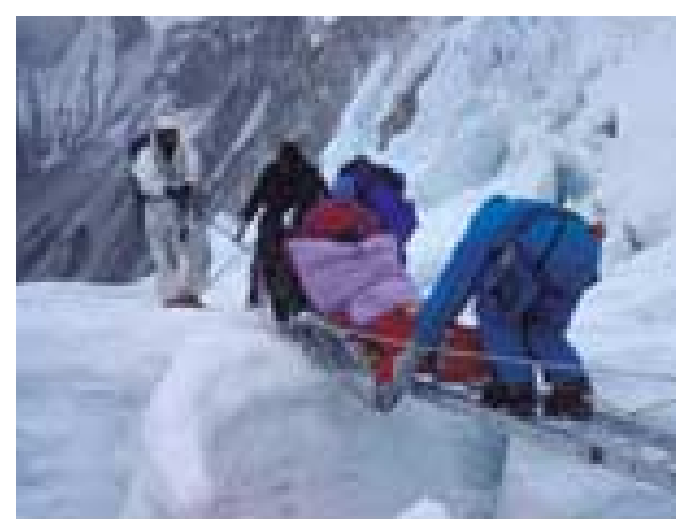

Introduction

Despite being one of the oldest threats to life and limb, cold exposure and consequent disorders are no less important in modern military practice as they were during classical times. Indeed, the desire of clothing providers to over-sell incremental improvements in protection may lead them to build unrealistic expectations, for example, claiming that a new boot will make cold injury a thing of the past (1). If only these conditions were that simple to prevent.

Cold-related illnesses result from the limited capacity of homeothermic humans to cope with cold exposure. The law of conservation of energy requires that, when heat loss exceeds heat production, there is a net loss of heat, reflected by a fall in tissue temperature. Physiological responses to whole body cold exposure include peripheral vasoconstriction, resulting in increased intrinsic insulation, and increased metabolic rate, particularly shivering. If the whole body is well protected but only a small portion of the periphery is cooled, then local vasoconstriction may be cyclically relieved by waves of cold-induced vasodilatation (2). However, in many circumstances the drive to preserve core temperature feeds a positive-feedback loop, in which vasoconstriction further lowers tissue temperatures, and local cold injury is the outcome (3). The adverse consequence of whole body cooling, hypothermia, is thus not infrequently concomitant with local freezing or nonfreezing cold injury of the peripheries.

This review encompasses the principal local conditions that result from cold exposure: freezing cold injury (which includes frostnip and frostbite), and nonfreezing cold injury (commonly called trench or immersion foot). Ill-defined conditions such as chilblains, and rarer disorders such as cold urticaria and erythrocyanosis, will not be covered.

\section{Freezing Cold Injury (Frostbite)}

The exact prevalence of frostbite is uncertain, and in the past the military accounted for the majority of cases. With increasing world trav$\mathrm{el}$, and more participants in skiing, mountaineering and other winter leisure pursuits there has been a steady rise in the prevalence amongst civilians. Non-freezing cold injuries occur at $0^{\circ}-15^{\circ} \mathrm{C}$, whilst freezing injuries occur below $0^{\circ} \mathrm{C}$ in dry conditions and this can be exacerbated by wet tissues, wind chill and altitude.

Frostnip is a reversible freezing cold injury which resolves completely within 30 minutes of starting to rewarm. Whilst clinically reversible, the subject remains at considerably greater risk of sustaining a further freezing cold injury for some months. If symptoms or signs persist beyond 30 minutes the subject had frostbite.

\section{Epidemiology}

In one study in the Finnish army, 1.8\% per 1,000 recruits suffered from frostbite (4). The prevalence of cold injury increases with falling temperature, and whilst no link was found between smoking and frostbite, previous cold injury has been shown to be a significant risk factor $(5,6)$.

\section{Risk factors}

A number of factors increase the risk of developing frostbite, including behavioural aspects such as the individual's clothing, consumption of alcohol and other drugs, and access to (and use of) appropriate shelter. Physiological factors such as dehydration, hypoxia, and high altitude also appear to increase the risk of developing frostbite. Certain medical conditions such as diabetes, peripheral vascular disease, Raynauds phenomenon, psychiatric illness and previous frostbite predispose to the condition. Certain mechanical factors may affect the risk of developing frostbite such as tight, wet or inadequate clothing, contact with a conducting material and immobility, and exposure to high winds. In most situations where frostbite develops more than one factor 


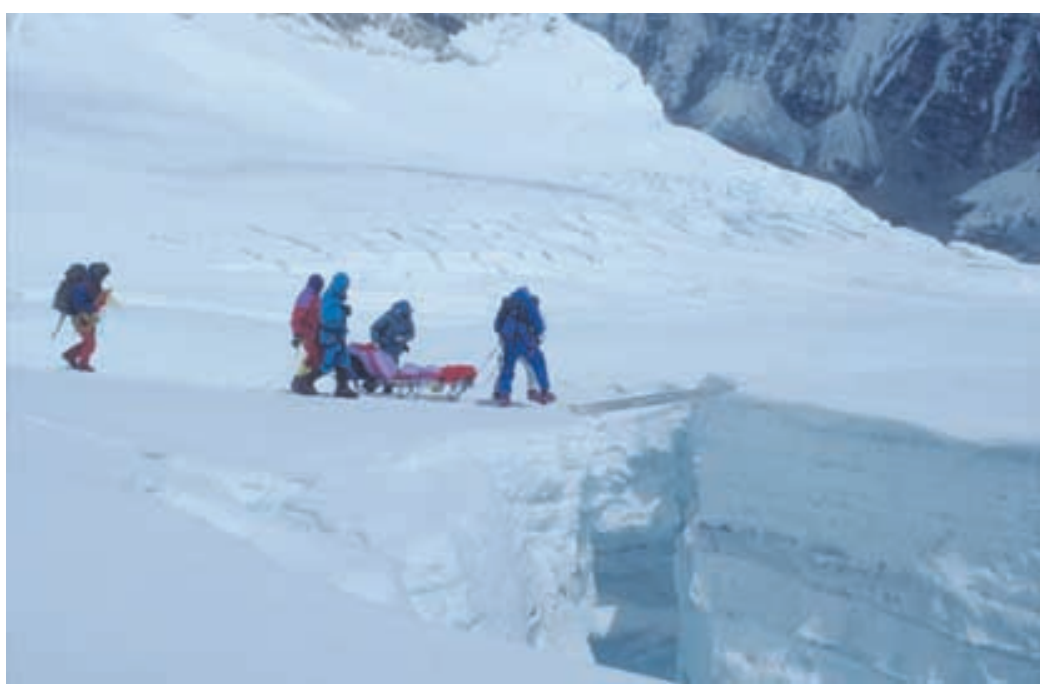

will be involved and each parameter will have a synergistic effect with the others.

\section{Pathophysiology}

Whilst any area may be affected, certain areas are more susceptible because of site, pressure, insulation or susceptibility to wetting. Examples include the face, tips of the nose and ears, fingers and hands, toes, feet and male genitalia.

\section{Classification}

There are four stages of frostbite:

Stage 1: Pre-freezing stage occurs before ice crystal forms $3-10^{\circ} \mathrm{C}$. Cutaneous sensation is lost below $10^{\circ} \mathrm{C}$.

Stage 2: Freeze-thaw phase occurs when tissue temperature is below $0^{\circ} \mathrm{C}$ and is a result of extra-cellular ice crystal formation. Environmental temperatures are usually $-6^{\circ}$ to $-15^{\circ} \mathrm{C}$.

Stage 3: Vascular stage results in coagulation within the vessels, plasma leakage and shunting.

Stage 4: Late ischaemic phase occurs with thrombosis, ischaemia, gangrene and loss of autonomic control.

\section{Field treatment}

\section{Prevention}

Prevention is the key. Awareness and experience are the keys to prevention.

\section{Diagnosis}

Diagnosis is usually straightforward but an awareness of the risk is important. Frostbite may be initially painful but may not be so. If a cold extremity becomes numb, frostbite is the likely cause. The skin may become white and blanched in appearance and tissues feel "woody" to the patient. If a large area becomes frostbitten it may become purple due to sludging of blood.

\section{Treatment}

Frostnip rapidly warmed should result in no long-term damage.
For frostbite:

1. Protect from further cold injury. Remove jewellery, put on dry gloves/mitts/socks if possible, adjust boots.

2. Do not deliberately thaw part until it can be rapidly rewarmed in a water bath in a situation from which evacuation can be arranged without further pressure on damaged tissue (normally base camp).

3. As soon as available give aspirin $300 \mathrm{mg}$ daily to facilitate circulation and inhibit potentially damaging toxins.

4. If above $4,000 \mathrm{~m}$ and if available, give oxygen.

5. On arrival at a secure base the patient should first be treated for potential hypothermia by slow rewarming with insulation in a warm environment.

6. The patient should be kept well hydrated with warm fluid drinks.

7. The frostbitten part should be rapidly rewarmed by immersion in warm water. This should be at $40^{\circ}-42^{\circ} \mathrm{C}$ (baby bath temperature). The bath should contain a large enough volume of water to minimise cooling by the cold body part and to facilitate easy top-ups with warm water to maintain the temperature. The damaged part should not be in contact with the side of the bath. Antiseptic can be added to the water if available. Full thawing may take an hour or more.

8. Thawing may be very painful and strong painkillers may be needed by injection.

9. Once thawed the frostbitten part must not be used. A patient with frostbitten toes or feet will have to be carried. A patient with frostbitten fingers or hands will be unable to use a walking stick, ice axe or crutch and will need help with dressing, eating and going to the toilet.

10. The damaged part should be loosely bandaged with dry protective nonadherent dressings and well padded, especially between digits. Try to avoid bursting blisters but if inevitable do so with sterile needle or blade.

11. Take daily digital photos and photos of all procedures for medical records and to facilitate e-mail transmission if advice is needed.

12. If there is any suspicion of infection start antibiotics.

13. Check patient is up to date with tetanus cover.

\section{Immediate hospital treatment \\ Rewarming}

In a hypothermic patient, whole body warming should take priority over the limb. Once 
the patient's core temperature has reached approximately $35^{\circ} \mathrm{C}$, limb warming can begin. Rewarming in a whirlpool bath (temperature $38^{\circ} \mathrm{C}$ ) containing dilute antiseptic solution should aim to warm the $\operatorname{limb}(\mathrm{s})$ over a twenty to sixty minute period. Adequate analgesia is essential.

\section{Imaging}

A number of different imaging modalities including ultrasound, Duplex, angiography and MRA have been used over the years, the aim being trying to try to predict the ultimate clinical outcome. Technitium ${ }^{99}$ bone scanning when performed at 48-72 hours currently appears to give the best prognostic information about likely outcome.

\section{Subsequent hospital treatment}

The next phase is lengthy. Aims should be to provide a restful positive environment focusing on all aspects of the individual's care. Frostbitten tissue should be regularly dressed, and should be elevated and rested. Evacuation from remote field units at this time is appropriate. Demarcation can take up to 90 days and it is important both the patient and team are aware of this period of apparent inactivity.

A number of adjunctive treatments have been studied with varying degrees of scientific rigour. The underlying aim of the adjunctive treatments is to improve the local circulation whilst at the same time limiting local tissue damage. Many of the therapies have been trialled in patients with peripheral vascular disease.

\section{Minor surgery}

Whilst local wound debridement and wound toilet is often required it should be limited. Amputations should normally be delayed until approximately 60-90 days.

\section{Hyperbaric oxygen treatment}

The role of hyperbaric oxygen treatment in frostbite is currently uncertain, with no level 1 evidence to support its use. A number of animal studies have demonstrated it is of little or no benefit (7) yet recent human studies have reported encouraging results $(8,9)$. Further study is clearly required.

\section{Sympathectomy}

The role of lumbar or thoracic sympathectomy has yielded mixed results. When performed within hours of the injury, there appears to be little or no benefit. However, a more delayed approach has been found to be beneficial. Open, chemical (lumbar) or endoscopic transthoracic sympathectomy has a role in preventing some of the longerterm sequelae of frostbite such as pain (often due to vasospasm), paraesthesia and hyperhidrosis.

\section{Vasodilators}

Iloprost is a prostacyclin analogue with profound vasodilatory properties and has been used with some success. It is used in arterial surgery to mimic the effect of a sympathectomy. Intra-arterial reserpine has been used in frostbite to prevent vasospasm. The use of pentoxifylline, a methyl-xanthine derived phosphodiesterase inhibitor, has been shown to enhance tissue survival.

\section{Amputation}

Failure to delay surgery is a major source of avoidable morbidity. There is rarely any urgency to intervene and so it should be undertaken by a surgeon with appropriate experience. The functional end result of any surgery needs to be considered and ideally where major limb loss is forseen the early involvement of a multidisciplinary rehabilitation team will result in better long-term functional results.

\section{Long-term sequelae}

The long-term sequelae of frostbite are less well studied. The functional use of extremities following a partial amputation is variable and injury specific. Major lower limb amputations, whilst rare, are occasionally necessary and appropriate multidisciplinary care is essential. Tissue that has recovered from frostbite is more susceptible to further injury and this needs to born in mind when advising individuals about a return to environments where they may be at risk. Preventative measures remain the mainstay to primary and secondary treatment.

Non-Freezing Cold Injury (NFCI)

In contrast to freezing cold injury, non-freezing injury occurs when tissue fluids do not freeze (at about $-0.5^{\circ} \mathrm{C}$ ), but local temperatures remain low for several hours or days. Clues provided in the history include the feet (the most common site of injury) having been cold and wet, but without the formation of ice crystals in the socks. The diagnostic criterion is the combination of exposure to suitable causative conditions, coupled with a local neuropathy. The latter is most commonly anaesthesia, but can be described as 'cotton wool feet', 'feeling as if wearing someone else's feet', or loss of proprioception causing difficulty in walking particularly over rough ground. This initial phase of injury is also marked by profound vasoconstriction, and termed Ungley Stage 1 after the Naval Surgeon who first delineated the sequence (10).

When the affected extremity is rewarmed, either on return to barracks at the end of an exercise or during foot-care routines in the field, a fleeting phase of pale cyanosis may be observed in Ungley Stage 2. Once fully rewarmed, Ungley Stage 3 is marked by hyperaemia, with redness, swelling, full pulses and onset of pain. This pain is much more prolonged than the rewarming pain normally 
experienced in freezing cold injury, and is a common reason for presentation. However, those who enter Stage 3 whilst still in the field may suffer such gross swelling as to be unable to put their boots back on; this can be catastrophic to military operations, and had a major influence on the Falklands Conflict (11).

Exposure to cold wet conditions is most common, but by no means requisite. NFCI appears to be part of a spectrum of conditions resulting from vascular stasis and ischaemia, followed by hyperaemia and pain (11). Paddy foot, which often includes rampant fungal infection as well, results from warm immersion for several days, and has had operational impact on several military campaigns in the tropics, including the Vietnam War (12). Shelter limb was described in Londoners who took refuge in the deep underground tunnels during the Blitz in the Second World War, and appears to have occurred largely as a result of immobility, dependency, and popliteal pressure from deck chairs. There are other similarities with atypical pain syndromes, and deprivation hands and feet, seen in infants living in under-heated housing during temperate winters.

Stage 3 can last up to 3 months after initial injury, during which lasting sequelae commonly develop. Sensitivity to cold exposure is frequent, even after minor or sub-clinical NFCI, and can be demonstrated using infrared thermography before and after a standardised mild cold stress, such as immersion of the extremity in $15^{\circ} \mathrm{C}$ water for 2 minutes (13). This can be so severe as to prevent activities such as shopping in the freezer sections of supermarkets, and treatment with sustainedrelease nifedipine is usually only beneficial in those most seriously affected. Avoidance of all cold exposure, by working indoors in heated buildings during the winter, for example, can allow this sensitivity to subside, although in some patients it lasts many years. Living and working in a tropically hot climate may be the best option, and anecdotally has led to complete clinical recovery after 2-3 years.

Pain is intractable except to amitriptyline (and presumably other tricyclic antidepressants), and as with post-herpetic neuralgia, if treatment is not instituted promptly, there is a significant risk of chronic pain that is resistant to all treatment modalities. Pain is most prominent at night, and typically occurs in the soles of the feet, at the base of the toes. Amitriptyline should initially be given in a single $50 \mathrm{mg}$ dose (for the average well-built infantry soldier) a couple of hours before retiring, and can be increased to a maximum of $150 \mathrm{mg}$ if necessary to bring it under control (11). Untreated, a small proportion (typically less than $5 \%$ ) of patients will suffer chronic pain. Unfortunately no form of sympathetic block can then be attempted, as that universally results in medium and long-term deterioration. It is thus essential to control pain fol- lowing NFCI at the earliest opportunity.

Other sequelae include hyperhidrosis, recurrent nail loss, persistent sensory disturbance, causalgia, allodynia, and chronic alteration of gait leading in turn to foot, knee, hip and back problems $(11,13,14)$. Although almost all cases of NFCI involve the feet, as many as $25 \%$ may also have injured hands, particularly in Afro-Caribbeans who have significantly increased susceptibility to NFCI as well as freezing cold injury (15).

Hyperaemia during Ungley Stage 3 appears to be an important part of the pathogenesis, probably in the generation of free radicals and vascular endothelial damage (11). Rapid rewarming, as would be accomplished by treating a non-freezing injury as if it had frozen, or inadvertently during routine postexercise hygiene, thus augments the hyperaemia and worsens outcome (16). Accordingly joint treatment policy (17) strongly recommends that NFCI should be allowed to re-warm slowly and not rapidly (as for freezing cold injury). It is possible that hyperbaric oxygen may have value in early treatment too, although no trials appear to have assessed that use. Gross tissue damage following NFCI is relatively rare in peacetime experience, and after initial slow rewarming, management should follow the standard conservative protocol employed in freezing injury.

With the likelihood of chronic sequelae and limited potential for treatment, the only effective approach to NFCI is to try to prevent its occurrence. This requires the enthusiastic engagement of command, trainers, and those at risk of sustaining injury. Detailed measures are given in JSP 539 Chapter 3 (17), together with full treatment protocols.

\section{Conclusion}

This review has examined the local effects of cold injury. Improvements in the treatment of both FCI and NFCI are largely dependent on further work with animal models, which have so far failed to deliver on their early promise. The management of those suffering from chronic pain remains very difficult, but should be a largely avoidable problem if cases are treated early with amitriptyline. As with other aspects of local cold injury, improved awareness among medical staff, command, trainers, and those at risk will bring greatest returns.

\section{References}

1. Oakley EHN. The design and function of military footwear: a review following experiences in the South Atlantic. Ergonomics 1984;27:631-7.

2. Daanen HAM. Finger cold-induced vasodilation: a review. Eur f Appl Physiol 2003;89:411-26.

3. Francis TJR, Golden FStC. Non-freezing cold injury: the pathogenesis. F Roy Nav Med Serv 1985;71:3-8.

4. Lehmuskallio $\mathrm{E}$, Linholm $\mathrm{H}$, Koskenvvo $\mathrm{K}$ et al. Frostbite of the face and ears: an epidemiological study of risk factors in Finnish conscripts. BMF 1995; 311:1661-3.

5. Sumner DS, Criblez T, Doolittle WH. Host factors in human frostbite. Mil Med 1974;139:454-61. 
6. Cattermole TJ. The epidemiology of cold injury in Antartica. Aviat Space Environ Med 1999;70:13540.

7. Murphy JV, Banwell PE, Roberts AHN, McGrouther AD. Frostbite: Pathogenesis and Treatment. F Trauma 2000; 48(1),171-178.

8. Heimburg D, Noah ME, Siekmann UPF, Pallua N. Hyperbaric oxygen treatment in deep frostbite of both hands in a boy. Burns 2001;27,404-408.

9. Finderle Z, Cankar K. Delayed treatment of frostbite injury with hyperbaric oxygen therapy: a case report. Av Space Env Med 2002;73(4),393-394.

10. Ungley CC, Blackwood W. Peripheral vasoneuropathy after chilling. 'Immersion foot and immersion hand'. Lancet 1942;2:447-51.

11. Thomas JR, Oakley EHN. Nonfreezing cold injury. In: Pandolf KB, Burr RE eds. Textbook of Military Medicine, Medical Aspects of Harsh Environments, vol 1. Washington DC: US Army, 2001;467-90.
12. Akers WA. Paddy foot: a warm water immersion foot syndrome variant. Part 1 . The natural disease, epidemiology. Mil Med 1974;139:605-12.

13. Oakley EHN. Cold injury. In: Tooke JE, Lowe GDO eds. A Textbook of Vascular Medicine. London: Arnold, 1996;353-70.

14. Oakley EHN. The Long-term Sequelae of Cold Injury Among 'The Chosin Few'. INM Report no. 96043. Gosport, UK: Institute of Naval Medicine, 1996.

15. Sumner DS, Criblez TL, Doolittle WH. Host factors in human frostbite. Mil Med 1974;141:454-61.

16. Smith JL, Ritchie J, Dawson J. On the pathology of trench frostbite. Lancet 1915;11 Sep:595-8.

17. Army Medical Directorate. Climatic Injuries in the Armed Forces: Prevention and Treatment. Joint Services Publication 539. London: Ministry of Defence, UK, 2003. 